Open J. Math. Sci., Vol. 2(2018), No. 1, pp. 307-322

Website: https://pisrt.org/psr-press/journals/oms/

ISSN: 2523-0212 (Online) 2616-4906 (Print)

http://dx.doi.org/10.30538/oms2018.0037

\title{
OSCILLATION CRITERIA FOR NONLINEAR DYNAMIC EQUATIONS ON TIME SCALES
}

\author{
MERVE ZINGIL, FATMA SERAP TOPAL ${ }^{1}$
}

\begin{abstract}
The main goal of this article is to study the oscillation criteria of the second-order neutral differential equations on time scales. We give several theorems and related examples to illustrate the applicability of these theorems. Our results extend some recent work in the literature.
\end{abstract}

Mathematics Subject Classification: 34K11, 39A10, 39A99.

Key words and phrases: neutral dynamic equations; oscillation; time scales; nonlinear delay dynamic. equation

\section{Introduction}

When delays appear in additional terms involving the highest order derivative of the unknown function in a differential equation, we are dealing with a neutral type differential equation. Neutral functional differential equations have numerous applications in electric networks. For instance, they are frequently used for the study of distributed networks containing lossless transmission lines which rise in high speed computers where the lossless transmission lines are used to interconnect switching circuits; see [1]. Recently, many results on oscillation of nonneutral differential equations and neutral functional differential equations have been established. We refer the reader to $[2,3,4]$ and the references cited therein.

The theory of time scales is initiated by Hilger $[5,6]$ in order to unify continuous and discrete analysis. Several authors have expounded on various aspects of the theory of dynamic equations on time scales; see the survey paper by Agarwal et al. [7] and the references cited therein. The books on the subject of time

Received 11 May 2018. Revised 20 October 2018.

1 Corresponding Author

(C) 2018 Merve Zingil, Fatma Serap Topal. This is an open access article distributed under the Creative Commons Attribution License, which permits unrestricted use, distribution, and reproduction in any medium, provided the original work is properly cited. 
scales, by Bohner and Peterson [8], summarize and organize much of time scale calculus. There are applications of dynamic equations on time scales to quantum mechanics, electrical engineering, neural networks, heat transfer, combinatorics, etc.

In recent years, there has been much research activity concerning the oscillation and nonoscillation of solutions of ordinary dynamic equations on time scales, we refer the reader to the papers $[9,10,11,12,13,14,15,16,17,18,19,20,21,22$, 23]. Recently Agarwal et al. [24] have established some new oscillation criteria for second-order delay dynamic equations on time scales.

Very recently, some authors studied on existence and behavior of solutions for some integral equations, second order multi objective symmetric programming problem and duality relations and fixed point theorems for nonlinear contractions in $[25,26,27,28,29]$.

In this paper, we consider second-order nonlinear neutral dynamic equations of the following form:

$$
\left[r(t)\left((m(t) y(t)+p(t) y(\tau(t)))^{\Delta}\right)^{\gamma}\right]^{\Delta}+f_{1}\left(t, y\left(\delta_{1}(t)\right)+f_{2}\left(t, y\left(\delta_{2}(t)\right)=0\right.\right.
$$

On a time scale $\mathbb{T}$ satisfying inf $\mathbb{T}=t_{0}$ and $\sup \mathbb{T}=\infty$. We recall that a solution of equation (1) is said to be oscillatory on $\left[t_{0}, \infty\right)_{\mathbb{T}}$ in case it is neither eventually positive nor eventually negative. Otherwise, it is said to be nonoscillatory. Equation (1) is said to be oscillatory in case all of its solutions are oscillatory. Throughout this paper, we assume the followings:

$\left(H_{1}\right) \tau(t), \delta_{i}(t) \in C_{r d}(\mathbb{T}, \mathbb{T})$ such that $\tau(t) \leqslant t$ and $\lim _{t \rightarrow \infty} \tau(t)=\lim _{t \rightarrow \infty} \delta_{i}(t)=$ $\infty, \quad i=1,2$,

$\left(H_{2}\right) r(t) \in C_{r d}\left(\mathbb{T}, \mathbb{R}^{+}\right)$such that $\int_{t_{0}}^{\infty} \frac{1}{r^{\frac{1}{\gamma}}(t)} \Delta t=\infty$ and $m(t) \in C_{r d}\left(\mathbb{T}, \mathbb{R}^{+}\right)$, $p(t) \in C_{r d}(\mathbb{T},[0,1))$ such that $m(\tau(t))>p(t)$ where $\mathbb{R}^{+}=[0, \infty)$,

$\left(H_{3}\right) f_{i}(t, u): \mathbb{T} \times \mathbb{R} \longrightarrow \mathbb{R}$ are continuous functions such that $u f_{i}(t, u)>0$ for all $u \neq 0$ and there exist $q_{i}(t) \in C_{r d}\left(\mathbb{T}, \mathbb{R}^{+}\right)(i=1,2), \gamma$ is quotients of odd positive integers $\alpha$ and $\beta$ with $0<\alpha \leq \beta$ such that $\left|u f_{1}(t, u)\right| \geq$ $q_{1}(t)|u|^{\alpha+1},\left|u f_{2}(t, u)\right| \geq q_{2}(t)|u|^{\beta+1}$.

This paper is organized as follows. After this introduction, we introduce some basic lemmas in Section 2. In Section 3, we present the main results and give an example to illustrate the main results.

\section{Some preliminaries}

Before stating our main results, we'll give some lemmas which play an important role in the proof of the main results. Set

$$
x(t):=m(t) y(t)+p(t) y(\tau(t))
$$

then the equation (1) becomes

$$
\left(r(t)\left(x^{\Delta}(t)\right)^{\gamma}\right)^{\Delta}+f_{1}\left(t, y\left(\delta_{1}(t)\right)+f_{2}\left(t, y\left(\delta_{2}(t)\right)=0 .\right.\right.
$$


For $t, T \in \mathbb{T}$ with $t>T$, we define

$$
\begin{gathered}
R(t, T)=\int_{T}^{t} \frac{1}{(r(s))^{\frac{1}{\gamma}}} \Delta s, \\
\beta(t, T)= \begin{cases}\frac{R\left(\delta_{i}(t), T\right)}{R(t, T)}, & \delta_{i}(t)<t ; \\
1, & \delta_{i}(t) \geq t,\end{cases} \\
\eta^{\sigma}(t)= \begin{cases}1, & \alpha=\gamma ; \\
c_{2}^{\alpha-\gamma}\left(\int_{T}^{\sigma(t)} \frac{1}{r^{\frac{1}{\gamma}}(s)} \Delta s\right)^{\alpha-\gamma}, & \alpha<\gamma ; \\
c_{1}, & \alpha>\gamma,\end{cases}
\end{gathered}
$$

and

$$
Q_{1}(t)=Q(t)\left(\frac{r^{\frac{1}{\gamma}}(t) R(t, T)}{r^{\frac{1}{\gamma}}(t) R(t, T)+\mu(t)}\right)^{\alpha} \eta^{\sigma}(t),
$$

where $Q(t)$ will be defined as Lemma 2.2. For $D=\left\{(t, s) \in \mathbb{T}^{2}: t \geq s \geq 0\right\}$, we define

$\mathcal{H}=\left\{H(t, s) \in C_{r d}^{1}(D,[0, \infty)): H(t, t)=0, H(t, s)>0 \quad\right.$ and $H_{s}^{\Delta}(t, s) \geq$ 0 for $t>s \geq 0\}$

and

$$
C(t, s)=H^{\Delta}(t, s) z(s)+H(t, \sigma(s)) z^{\Delta}(s) \text { for } H(t, s) \in \mathcal{H}
$$

where $z \in C_{r d}^{1}(\mathbb{T},(0, \infty))$ is to be given Theorem 3.2 and Theorem 3.3, and $z_{+}^{\Delta}(t)=\max \left\{z^{\Delta}(t), 0\right\}$.

Lemma 2.1. Assume that $\left(H_{1}\right)-\left(H_{3}\right)$ and (1) are satisfied. If equation (1) has a nonoscillatory solutions $y$ on $\left[t_{0}, \infty\right)_{\mathbb{T}}$, and $x$ is defined as in (2), then there exists a $T \in \mathbb{T}$ sufficiently large such that $x(t)>0, x^{\Delta}(t)>0,\left(r(t)\left(x^{\Delta}(t)\right)^{\gamma}\right)^{\Delta}<$ $0, x(t) \geq r^{\frac{1}{\gamma}}(t) x^{\Delta}(t) R(t, T), x\left(\delta_{i}(t)\right) \geq \beta(t, T) x(t)$ for $t \in[T, \infty)_{\mathbb{T}}$.

Proof. If $y(t)$ is an eventually positive solution of (1), then there exist a $T \in$ $\left[t_{0}, \infty\right)_{\mathbb{T}}$ such that

$$
y(t)>0, y(\tau(t))>0, \quad y\left(\delta_{i}(t)\right)>0, \text { for } t \geq T, \quad i=1,2 .
$$

From (2), (4) and $\left(H_{2}\right), x(t)>0$. Also by $(3)$ and $\left(H_{3}\right)$, we have

$$
\begin{aligned}
\left(r(t)\left(x^{\Delta}(t)\right)^{\gamma}\right)^{\Delta} & =-f_{1}\left(t, y\left(\delta_{1}(t)\right)-f_{2}\left(t, y\left(\delta_{2}(t)\right)\right.\right. \\
& \leq-q_{1}(t) y^{\alpha}\left(\delta_{1}(t)\right)-q_{2}(t) y^{\beta}\left(\delta_{2}(t)\right)<0, \text { for } t \geq T,
\end{aligned}
$$

which implies that $r(t)\left(x^{\Delta}(t)\right)^{\gamma}$ is decreasing on $[T, \infty)_{\mathbb{T}}$.

We claim that $r(t)\left(x^{\Delta}(t)\right)^{\gamma}>0$ on $[T, \infty)_{\mathbb{T}}$. Assume not, there is a $t_{1} \in[T, \infty)_{\mathbb{T}}$ such that $r\left(t_{1}\right)\left(x^{\Delta}\left(t_{1}\right)\right)^{\gamma}=c<0$. Since $r(t)\left(x^{\Delta}(t)\right)^{\gamma}$ is decreasing on $[T, \infty)_{\mathbb{T}}$, $r(t)\left(x^{\Delta}(t)\right)^{\gamma} \leq r\left(t_{1}\right)\left(x^{\Delta}\left(t_{1}\right)\right)^{\gamma}=c$ for $t \geq t_{1}$. So, we have

$$
x^{\Delta}(t) \leq \frac{c^{\frac{1}{\gamma}}}{r^{\frac{1}{\gamma}}(t)} .
$$


Integrating the above inequality from $t_{1}$ to $t$, by $\left(H_{2}\right)$, we get

$$
x(t) \leq x\left(t_{1}\right)+c^{\frac{1}{\gamma}} \int_{t_{1}}^{t} \frac{1}{r^{\frac{1}{\gamma}}(s)} \Delta s \rightarrow-\infty \quad(t \rightarrow \infty)
$$

and this contradicts the fact that $x(t)>0$ for all $t \geq T$. Thus, we have $r(t)\left(x^{\Delta}(t)\right)^{\gamma}>0$ on $[T, \infty)_{\mathbb{T}}$ and so $x^{\Delta}(t)>0$ on $[T, \infty)_{\mathbb{T}}$. From $r(t)\left(x^{\Delta}(t)\right)^{\gamma}$ is decreasing on $[T, \infty)_{\mathbb{T}}$, we have

$$
\begin{aligned}
x(t) & =x(T)+\int_{T}^{t} x^{\Delta}(s) \Delta s \\
& =x(T)+\int_{T}^{t} \frac{\left(r(s)\left(x^{\Delta}(s)\right)^{\gamma}\right)^{\frac{1}{\gamma}}}{r^{\frac{1}{\gamma}}(s)} \Delta s \\
& >\int_{T}^{t} \frac{\left(r(s)\left(x^{\Delta}(s)\right)^{\gamma}\right)^{\frac{1}{\gamma}}}{r^{\frac{1}{\gamma}}(s)} \Delta s \\
& >\left(r(t)\left(x^{\Delta}(t)\right)^{\gamma}\right)^{\frac{1}{\gamma}} \int_{T}^{t} \frac{1}{r^{\frac{1}{\gamma}}(s)} \Delta s \\
& =r^{\frac{1}{\gamma}}(t) x^{\Delta}(t) R(t, T) .
\end{aligned}
$$

Now, we will show that

$$
x\left(\delta_{i}(t)\right) \geq \beta(t, T) x(t) .
$$

We consider two cases which $\delta_{i}(t)<t$ and $\delta_{i}(t) \geq t$, respectively.

Case 1. $\delta_{i}(t)<t$. Since $r(t)\left(x^{\Delta}(t)\right)^{\gamma}$ is decreasing on $[T, \infty)_{\mathbb{T}}$, we have

$$
\begin{aligned}
x(t)-x\left(\delta_{i}(t)\right) & =\int_{\delta_{i}(t)}^{t} \frac{\left(r(s)\left(x^{\Delta}(s)\right)^{\gamma}\right)^{\frac{1}{\gamma}}}{r^{\frac{1}{\gamma}}(s)} \Delta s \\
& \leq\left(r\left(\delta_{i}(t)\right)\left(x^{\Delta}\left(\delta_{i}(t)\right)\right)^{\gamma}\right)^{\frac{1}{\gamma}} \int_{\delta_{i}(t)}^{t} \frac{1}{r^{\frac{1}{\gamma}}(s)} \Delta s .
\end{aligned}
$$

By dividing $x\left(\delta_{i}(t)\right)$, it follows that

$$
\frac{x(t)}{x\left(\delta_{i}(t)\right)} \leq 1+\frac{\left(r\left(\delta_{i}(t)\right)\left(x^{\Delta}\left(\delta_{i}(t)\right)\right)^{\gamma}\right)^{\frac{1}{\gamma}}}{x\left(\delta_{i}(t)\right)} \int_{\delta_{i}(t)}^{t} \frac{1}{r^{\frac{1}{\gamma}}(s)} \Delta s .
$$

Since $\delta_{i}(t) \geq T$ for $t \in[T, \infty)_{\mathbb{T}}$, we have

$x\left(\delta_{i}(t)\right) \geq \int_{T}^{\delta_{i}(t)} \frac{\left(r(s)\left(x^{\Delta}(s)\right)^{\gamma}\right)^{\frac{1}{\gamma}}}{r^{\frac{1}{\gamma}}(s)} \Delta s \geq\left(r\left(\delta_{i}(t)\right)\left(x^{\Delta}\left(\delta_{i}(t)\right)\right)^{\gamma}\right)^{\frac{1}{\gamma}} \int_{T}^{\delta_{i}(t)} \frac{1}{r^{\frac{1}{\gamma}}(s)} \Delta s$,

which implies that

$$
\frac{\left(r\left(\delta_{i}(t)\right)\left(x^{\Delta}\left(\delta_{i}(t)\right)\right)^{\gamma}\right)^{\frac{1}{\gamma}}}{x\left(\delta_{i}(t)\right)} \leq \frac{1}{\int_{T}^{\delta_{i}(t)} \frac{1}{r^{\frac{1}{\gamma}}(s)} \Delta s}
$$


Thus, we get

$$
\frac{x(t)}{x\left(\delta_{i}(t)\right)} \leq 1+\frac{\int_{\delta_{i}(t)}^{t} \frac{1}{r^{\frac{1}{\gamma}}(s)} \Delta s}{\int_{T}^{\delta_{i}(t)} \frac{1}{r^{\frac{1}{\gamma}}(s)} \Delta s}=\frac{\int_{T}^{t} \frac{1}{r^{\frac{1}{\gamma}}(s)} \Delta s}{\int_{T}^{\delta_{i}(t)} \frac{1}{r^{\frac{1}{\gamma}}(s)} \Delta s}=\frac{R(t, T)}{R\left(\delta_{i}(t), T\right)} .
$$

So,

$$
x\left(\delta_{i}(t)\right) \geq \beta(t, T) x(t) .
$$

Case 2. $\delta_{i}(t) \geq t$. Since $x^{\Delta}(t) \geq 0$ for $t \in[T, \infty)_{\mathbb{T}}, \quad x\left(\delta_{i}(t)\right) \geq x(t)$. From the definition of $\beta(t, T)$, we obtain

$$
x\left(\delta_{i}(t)\right) \geq \beta(t, T) x(t) .
$$

Lemma 2.2. Assume that conditions $\left(H_{1}\right)-\left(H_{3}\right)$ and (1) are satisfied. If equation (1) has a nonoscillatory solution $y$ on $\left[t_{0}, \infty\right)_{\mathbb{T}}$ and $x$ is defined as in (2) then there exists a $T>t_{0}$ such that

$$
\left(r(t)\left(x^{\Delta}(t)\right)^{\gamma}\right)^{\Delta}+Q(t) x^{\alpha}(t) \leq 0 \text { for } t \geq T
$$

where

$$
\begin{aligned}
Q(t)= & q_{1}(t)\left(\frac{1}{m\left(\delta_{1}(t)\right)}\left[1-\frac{p\left(\delta_{1}(t)\right)}{m\left(\tau\left(\delta_{1}(t)\right)\right)}\right]\right)^{\alpha} \beta^{\alpha}(t, T) \\
& +q_{2}(t)\left(\frac{1}{m\left(\delta_{2}(t)\right)}\left[1-\frac{p\left(\delta_{2}(t)\right)}{m\left(\tau\left(\delta_{2}(t)\right)\right)}\right]\right)^{\beta} \beta^{\beta}(t, T)>0 .
\end{aligned}
$$

Proof. If $y(t)$ is an eventually positive solution of (1) then there exists a $T \in$ $\left[t_{0}, \infty\right)_{\mathbb{T}}$ such that

$$
\begin{aligned}
& y(t)>0, \quad y(\tau(t))>0, \quad y\left(\delta_{i}(t)\right)>0, \quad y(\tau(\tau(t)))>0 \quad \text { for } t \geq T, \quad i=1,2 . \\
& y(t)=\frac{1}{m(t)}[x(t)-p(t) y(\tau(t))] \\
&=\frac{1}{m(t)}\left[x(t)-p(t) \frac{1}{m(\tau(t))}[x(\tau(t))-p(\tau(t)) y(\tau(\tau(t)))]\right. \\
& \geq \frac{1}{m(t)}\left[x(t)-p(t) \frac{1}{m(\tau(t))} x(\tau(t))\right] \\
& \geq \frac{1}{m(t)}\left[1-\frac{p(t)}{m(\tau(t))}\right] x(t) .
\end{aligned}
$$

For $t \geq t_{2}=\delta_{i}^{-1}(T)$ with $i=1,2$, noting that (5), we get

$$
y\left(\delta_{i}(t)\right) \geq \frac{1}{m\left(\delta_{i}(t)\right)}\left[1-\frac{p\left(\delta_{i}(t)\right)}{m\left(\tau\left(\delta_{i}(t)\right)\right)}\right] x\left(\delta_{i}(t)\right) .
$$

From Lemma 2.1 and (6), we have

$$
\left(r(t)\left(x^{\Delta}(t)\right)^{\gamma}\right)^{\Delta} \leq-q_{1}(t) y^{\alpha}\left(\delta_{1}(t)\right)-q_{2}(t) y^{\beta}\left(\delta_{2}(t)\right)
$$




$$
\begin{aligned}
\leq & -q_{1}(t)\left(\frac{1}{m\left(\delta_{1}(t)\right)}\left[1-\frac{p\left(\delta_{1}(t)\right)}{m\left(\tau\left(\delta_{1}(t)\right)\right.}\right]\right)^{\alpha} x^{\alpha}\left(\delta_{1}(t)\right) \\
& -q_{2}(t)\left(\frac{1}{m\left(\delta_{2}(t)\right)}\left[1-\frac{p\left(\delta_{2}(t)\right)}{m\left(\tau\left(\delta_{2}(t)\right)\right.}\right]\right)^{\beta} x^{\beta}\left(\delta_{2}(t)\right) \\
\leq & -q_{1}(t)\left(\frac{1}{m\left(\delta_{1}(t)\right)}\left[1-\frac{p\left(\delta_{1}(t)\right)}{m\left(\tau\left(\delta_{1}(t)\right)\right)}\right]\right)^{\alpha} \beta^{\alpha}(t, T) x^{\alpha}(t) \\
& -q_{2}(t)\left(\frac{1}{m\left(\delta_{2}(t)\right)}\left[1-\frac{p\left(\delta_{2}(t)\right)}{m\left(\tau\left(\delta_{2}(t)\right)\right)}\right]\right)^{\beta} \beta^{\beta}(t, T) x^{\beta}(t) \\
= & -q_{1}(t)\left(\frac{1}{m\left(\delta_{1}(t)\right)}\left[1-\frac{p\left(\delta_{1}(t)\right)}{m\left(\tau\left(\delta_{1}(t)\right)\right)}\right]\right)^{\alpha} \beta^{\alpha}(t, T) \\
& \left.-q_{2}(t)\left(\frac{1}{m\left(\delta_{2}(t)\right)}\left[1-\frac{p\left(\delta_{2}(t)\right)}{m\left(\tau\left(\delta_{2}(t)\right)\right.}\right]\right)^{\beta} \beta^{\beta}(t, T)\right] x^{\alpha}(t) .
\end{aligned}
$$

Thus,

$$
\left(r(t)\left(x^{\Delta}(t)\right)^{\gamma}\right)^{\Delta}+Q(t) x^{\alpha}(t) \leq 0 .
$$

Lemma 2.3. [9] Let $g(u)=B u-A u^{\frac{\gamma+1}{\gamma}}$, where $A>0$ and $B$ are constants, $\gamma$ is a quotient of odd positive integers. Then $g$ attains its maximum value on $\mathbb{R}$ at $u^{*}=\left(\frac{B \gamma}{A(\gamma+1)}\right)^{\gamma}$ and

$$
\max _{u \in \mathbb{R}} g\left(u^{*}\right)=\frac{\gamma^{\gamma}}{(\gamma+1)^{\gamma+1}} \frac{B^{\gamma+1}}{A^{\gamma}} .
$$

\section{Main Results}

In this section, we state and prove the main oscillation results for the equations (1).

Theorem 3.1. Assume that $\left(H_{1}\right)-\left(H_{3}\right)$ and (1) are satisfied. If

$$
\int_{t_{0}}^{\infty} Q_{1}(s) \Delta s=\infty
$$

then every solution of (1) oscillates.

Proof. Assume the contrary and let $y$ be a nonoscillatory solution of (1). Without loss of generality, we may assume that

$y(t)>0, \quad y(\tau(t))>0, \quad y(\tau(\tau(t)))>0, \quad y\left(\delta_{i}(t)\right)>0, \quad i=1,2$ for $t \geq T .(10)$

We define

$$
w(t):=\frac{x^{[1]}(t)}{x^{\gamma}(t)} \text { for } t \geq T,
$$


where

$$
x^{[1]}(t):=\left(r(t)\left(x^{\Delta}(t)\right)^{\gamma}\right)(t) \quad \text { and } \quad x^{[2]}(t):=\left(x^{[1]}(t)\right)^{\Delta} .
$$

Then, $w(t)>0$ for $t \geq T$. Since Lemma 2.1 and (2), there exists a $T \geq t_{0}$ such that

$$
x(t)>0, \quad x^{[1]}(t)>0 \text { and } x^{[2]}(t)<0 \text { for } t \geq T .
$$

From Lemma 2.2, we get

$$
\begin{aligned}
w^{\Delta}(t) & =\frac{x^{[2]}(t) x^{\gamma}(t)-\left(x^{\gamma}(t)\right)^{\Delta} x^{[1]}(t)}{x^{\gamma}(t)\left(x^{\sigma}(t)\right)^{\gamma}} \\
& \leq-Q(t) \frac{x^{\alpha}(t)}{\left(x^{\sigma}(t)\right)^{\gamma}}-\frac{\left(x^{\gamma}(t)\right)^{\Delta} x^{[1]}(t)}{x^{\gamma}(t)\left(x^{\sigma}(t)\right)^{\gamma}}
\end{aligned}
$$

By the Ptzsche chain rule, if $x^{\Delta}(t)>0$ and $\gamma>1$, then we get

$$
\begin{aligned}
\left(x^{\gamma}(t)\right)^{\Delta} & =\gamma \int_{0}^{1}\left[x(t)+\mu(t) h x^{\Delta}(t)\right]^{\gamma-1} x^{\Delta}(t) d h \\
& =\gamma \int_{0}^{1}\left[(1-h) x(t)+h x^{\sigma}(t)\right]^{\gamma-1} x^{\Delta}(t) d h \\
& \geq \gamma \int_{0}^{1}(x(t))^{\gamma-1} x^{\Delta}(t) d h \\
& =\gamma(x(t))^{\gamma-1} x^{\Delta}(t) .
\end{aligned}
$$

Again by the Ptzsche chain rule, if $x^{\Delta}(t)>0$ and $0<\gamma \leq 1$, then we have

$$
\begin{aligned}
\left(x^{\gamma}(t)\right)^{\Delta} & =\gamma \int_{0}^{1}\left[x(t)+\mu(t) h x^{\Delta}(t)\right]^{\gamma-1} x^{\Delta}(t) d h \\
& =\gamma \int_{0}^{1}\left[(1-h) x(t)+h x^{\sigma}(t)\right]^{\gamma-1} x^{\Delta}(t) d h \\
& \geq \gamma \int_{0}^{1}\left(x^{\sigma}(t)\right)^{\gamma-1} x^{\Delta}(t) d h \\
& =\gamma\left(x^{\sigma}(t)\right)^{\gamma-1} x^{\Delta}(t) .
\end{aligned}
$$

Since $x(t)$ is increasing and $x^{[1]}(t)$ is decreasing, for $\gamma>1$, we get

$$
\begin{aligned}
\frac{\left((x(t))^{\gamma}\right)^{\Delta} x^{[1]}(t)}{x^{\gamma}(t)\left(x^{\sigma}(t)\right)^{\gamma}} & \geq \frac{\gamma(x(t))^{\gamma-1} x^{\Delta}(t)\left(x^{[1]}(t)\right)^{\sigma}}{x^{\gamma}(t)\left(x^{\sigma}(t)\right)^{\gamma}} \\
& \geq \frac{\gamma\left(x^{[1]}(t)\right)^{\frac{1}{\gamma}}\left(x^{[1]}(t)\right)^{\sigma}}{r^{\frac{1}{\gamma}}(t) x(\sigma(t))(x(\sigma(t)))^{\gamma}} \\
& \geq \frac{\gamma\left(\left(x^{[1]}(t)\right)^{\sigma}\right)^{\frac{1}{\gamma}}\left(x^{[1]}(t)\right)^{\sigma}}{r^{\frac{1}{\gamma}}(t) x(\sigma(t))(x(\sigma(t)))^{\gamma}}
\end{aligned}
$$




$$
=\gamma \frac{1}{r^{\frac{1}{\gamma}}(t)}\left(w^{\sigma}(t)\right)^{\frac{1}{\gamma}+1} .
$$

Also for $0<\gamma \leq 1$, we have

$$
\begin{aligned}
\frac{\left((x(t))^{\gamma}\right)^{\Delta} x^{[1]}(t)}{x^{\gamma}(t)\left(x^{\sigma}(t)\right)^{\gamma}} & \geq \frac{\gamma(x(\sigma(t)))^{\gamma-1} x^{\Delta}(t)\left(x^{[1]}(t)\right)^{\sigma}}{x^{\gamma}(t)\left(x^{\sigma}(t)\right)^{\gamma}} \\
& \geq \frac{\gamma\left(x^{[1]}(t)\right)^{\frac{1}{\gamma}}\left(x^{[1]}(t)\right)^{\sigma}}{r^{\frac{1}{\gamma}}(t) x(\sigma(t))(x(\sigma(t)))^{\gamma}} \\
& \geq \frac{\gamma\left(\left(x^{[1]}(t)\right)^{\sigma}\right)^{\frac{1}{\gamma}}\left(x^{[1]}(t)\right)^{\sigma}}{r^{\frac{1}{\gamma}}(t) x(\sigma(t))(x(\sigma(t)))^{\gamma}} \\
& =\gamma \frac{1}{r^{\frac{1}{\gamma}}(t)}\left(w^{\sigma}(t)\right)^{\frac{1}{\gamma}+1} .
\end{aligned}
$$

Together (14) and (15), we obtain

$$
\frac{\left((x(t))^{\gamma}\right)^{\Delta} x^{[1]}(t)}{x^{\gamma}(t)\left(x^{\sigma}(t)\right)^{\gamma}} \geq \gamma \frac{1}{r^{\frac{1}{\gamma}}(t)}\left(w^{\sigma}(t)\right)^{\frac{1}{\gamma}+1} \quad \text { for } \gamma>0 .
$$

Substituting (16) in (13), we get

$$
w^{\Delta}(t) \leq-Q(t) \frac{x^{\alpha}(t)}{\left(x(\sigma(t))^{\gamma}\right.}-\gamma \frac{1}{r^{\frac{1}{\gamma}}(t)}\left(w^{\sigma}(t)\right)^{\frac{1}{\gamma}+1} .
$$

Since $x^{\sigma}(t)=x(t)+\mu(t) x^{\Delta}(t)$, we have

$$
\frac{x^{\sigma}(t)}{x(t)}=1+\mu(t) \frac{x^{\Delta}(t)}{x(t)}=1+\frac{\mu(t)}{r^{\frac{1}{\gamma}}(t)} \frac{\left(x^{[1]}(t)\right)^{\frac{1}{\gamma}}}{x(t)} .
$$

Since $x^{[1]}(t)$ is decreasing, we get

$$
\begin{aligned}
x(t) & =x(T)+\int_{T}^{t}\left(x^{[1]}(s)\right)^{\frac{1}{\gamma}}\left(\frac{1}{r(s)}\right)^{\frac{1}{\gamma}} \Delta s \\
& >\left(x^{[1]}(t)\right)^{\frac{1}{\gamma}} \int_{T}^{t}\left(\frac{1}{r(s)}\right)^{\frac{1}{\gamma}} \Delta s .
\end{aligned}
$$

Therefore,

$$
\frac{x(t)}{\left(x^{[1]}(t)\right)^{\frac{1}{\gamma}}} \geq \int_{T}^{t}\left(\frac{1}{r(s)}\right)^{\frac{1}{\gamma}} \Delta s=R(t, T), \text { for } t \geq T .
$$

Thus we have

$$
\frac{x^{\sigma}(t)}{x(t)}=1+\frac{\mu(t)}{r^{\frac{1}{\gamma}}(t)} \frac{\left(x^{[1]}(t)\right)^{\frac{1}{\gamma}}}{x(t)} \leq \frac{r^{\frac{1}{\gamma}}(t) R(t, T)+\mu(t)}{r^{\frac{1}{\gamma}}(t) R(t, T)} \text { for } t \geq T
$$

and so

$$
\frac{x(t)}{x^{\sigma}(t)} \geq \frac{r^{\frac{1}{\gamma}}(t) R(t, T)}{r^{\frac{1}{\gamma}}(t) R(t, T)+\sigma(t)-t} \text { for } t \geq T
$$


Thus, for $t \geq T$, we get

$$
\begin{aligned}
\frac{x^{\alpha}(t)}{(x(\sigma(t)))^{\gamma}} & =\left(\frac{x(t)}{x(\sigma(t))}\right)^{\alpha} \frac{1}{(x(\sigma(t)))^{\gamma-\alpha}} \\
& \geq\left[\frac{r^{\frac{1}{\gamma}}(t) R(t, T)}{r^{\frac{1}{\gamma}}(t) R(t, T)+\mu(t)}\right]^{\alpha}(x(\sigma(t)))^{\alpha-\gamma} .
\end{aligned}
$$

Consider following cases.

Case 1. Let $\alpha<\gamma$. Since $x^{[1]}(t)$ is positive and decreasing, it follows from Lemma 2.1 that $x^{[1]}(t) \leq x^{[1]}(T)=c$ for $t \geq T$. So,

$$
x^{\Delta}(t) \leq \frac{c^{\frac{1}{\gamma}}}{r^{\frac{1}{\gamma}}(t)} .
$$

This implies that,

$$
\begin{aligned}
x(\sigma(t)) & =x\left(t_{2}\right)+\int_{T}^{\sigma(t)} x^{\Delta}(s) \Delta s \\
& \leq x\left(t_{2}\right)+c^{\frac{1}{\gamma}} \int_{T}^{\sigma(t)} \frac{1}{r^{\frac{1}{\gamma}}(s)} \Delta s .
\end{aligned}
$$

Since $\alpha<\gamma$, we get

$$
x^{\alpha-\gamma}(t)>c_{2}^{\alpha-\gamma}\left(\int_{T}^{\sigma(t)} \frac{1}{r^{\frac{1}{\gamma}}(s)} \Delta s\right)^{\alpha-\gamma},
$$

where

$$
c_{2}=\left(\frac{1}{c}\right)^{\gamma} .
$$

Case 2. Let $\alpha=\gamma$. So, $\left(x^{\sigma}(t)\right)^{\alpha-\gamma}=1$.

Case 3. Let $\alpha>\gamma$. In this case, since $x^{\Delta}(t)>0$, there exist $t_{2} \geq t_{1}$ such that $x^{\sigma}(t)>x(t)>c>0$. This implies that $\left(x^{\sigma}(t)\right)^{\alpha-\gamma}>c_{1}$, where $c_{1}=c^{\alpha-\gamma}$.

Combining these three cases and using the definition of $\eta^{\sigma}$, we conclude that

$$
\left(x^{\sigma}(t)\right)^{\alpha-\gamma} \geq \eta^{\sigma}(t) .
$$

Hence,

$$
\frac{x^{\alpha}(t)}{(x(\sigma(t)))^{\gamma}} \geq\left(\frac{r^{\frac{1}{\gamma}}(t) R(t, T)}{r^{\frac{1}{\gamma}}(t) R(t, T)+\mu(t)}\right)^{\alpha} \eta^{\sigma}(t) .
$$

Substituting (25) to (17), we obtain,

$$
\begin{gathered}
w^{\Delta}(t) \leq-Q(t)\left(\frac{r^{\frac{1}{\gamma}}(t) R(t, T)}{r^{\frac{1}{\gamma}}(t) R(t, T)+\mu(t)}\right)^{\alpha} \eta^{\sigma}(t)-\gamma \frac{1}{r^{\frac{1}{\gamma}}(t)}\left(w^{\sigma}(t)\right)^{\frac{1}{\gamma}+1}, \\
w^{\Delta}(t)+Q_{1}(t)+\gamma \frac{1}{r^{\frac{1}{\gamma}}(t)}\left(w^{\sigma}(t)\right)^{\frac{1}{\gamma}+1} \leq 0
\end{gathered}
$$


and so

$$
-w^{\Delta}(t) \geq Q_{1}(t)+\frac{\gamma}{r^{\frac{1}{\gamma}}(t)}\left(w^{\sigma}(t)\right)^{1+\frac{1}{\gamma}}>Q_{1}(t), \text { for } t \geq T .
$$

It follows from the definition of $x^{[1]}(t)$ that

$$
x^{\Delta}(t)=\left(\frac{x^{[1]}(t)}{r(t)}\right)^{\frac{1}{\gamma}} .
$$

Integrating (28) from $T$ to $t$, we obtain

$$
x(t)=x(T)+\int_{T}^{t}\left(\frac{1}{r(s)} x^{[1]}(s)\right)^{\frac{1}{\gamma}} \Delta s, \text { for } t \geq T .
$$

Taking into account that $x^{[1]}(t)$ is positive and decreasing, we get

$$
x(t) \geq x(T)+\left(x^{[1]}(t)\right)^{\frac{1}{\gamma}} \int_{T}^{t} \frac{1}{r^{\frac{1}{\gamma}}(s)} \Delta s \text { for } t \geq T .
$$

Thus, we have

$$
\frac{\left(x^{[1]}(t)\right)^{\frac{1}{\gamma}}}{x(t)} \leq\left(\int_{T}^{t} \frac{1}{r^{\frac{1}{\gamma}}(s)} \Delta s\right)^{-1}
$$

and

$$
w(t)=\frac{x^{[1]}(t)}{x^{\gamma}(t)} \leq\left(\int_{T}^{t} \frac{1}{r^{\frac{1}{\gamma}}(s)} \Delta s\right)^{-\gamma} \text { for } t \in[T, \infty)_{\mathbb{T}},
$$

which implies, in view of $\left(H_{2}\right)$, that

$$
\lim _{t \rightarrow \infty} w(t)=0 .
$$

Integrating (27) from $T$ to $\infty$ and using the fact that $\lim _{t \rightarrow \infty} w(t)=0$ we obtain

$$
w(T) \geq \int_{T}^{\infty} Q_{1}(s) \Delta s .
$$

which contradicts (9). The proof is completed.

Theorem 3.2. Assume that $\left(H_{1}\right)-\left(H_{3}\right)$ and (1) are satisfied. If there exists a positive rd-continuous $\Delta$-differentiable function $z(t)$ such that

$$
\lim _{t \rightarrow \infty} \sup \int_{T}^{t}\left[z(s) Q_{1}(s)-\frac{r(s)\left(z^{\Delta}(s)\right)^{\gamma+1}}{(\gamma+1)^{\gamma+1} z^{\gamma}(s)}\right]=\infty,
$$

then every solution of (1) oscillates.

Proof. Assume the contrary and let $y$ be a nonoscillatory solution of (1). Without loss of generality, we may assume that

$y(t)>0, \quad y(\tau(t))>0, \quad y(\tau(\tau(t)))>0, \quad y\left(\delta_{i}(t)\right)>0, \quad i=1,2 \quad$ for $t \geq T .(31)$ 
Let $w$ be defined as in (11), then $w(t)>0$ for $t \geq T$. Using (26), the following inequality is true:

$$
w^{\Delta}(t) \leq-Q_{1}(t)-\gamma \frac{1}{r^{\frac{1}{\gamma}}(t)}\left(w^{\sigma}(t)\right)^{\frac{1}{\gamma}+1} \quad \text { for } t \geq T .
$$

Multiplying (32) by $z(t)$ and integrating from $T$ to $t$, we obtain

$$
\int_{T}^{t} z(s) Q_{1}(s) \Delta s \leq-\int_{T}^{t} z(s) w^{\Delta}(s) \Delta s-\int_{T}^{t} \frac{\gamma z(s)}{r^{\frac{1}{\gamma}}(s)}\left(w^{\sigma}(s)\right)^{\frac{1}{\gamma}+1} \Delta s .
$$

Integration by parts, we get

$$
\begin{aligned}
-\int_{T}^{t} z(s) w^{\Delta}(s) \Delta s & =-z(t) w(t)+z(T) w(T)+\int_{T}^{t} z^{\Delta}(s) w^{\sigma}(s) \Delta s \\
& \leq z(T) w(T)+\int_{T}^{t} z^{\Delta}(s) w^{\sigma}(s) \Delta s .
\end{aligned}
$$

It follows that

$$
\int_{T}^{t} z(s) Q_{1}(s) \leq z(T) w(T)+\int_{T}^{t} z^{\Delta}(s) w^{\sigma}(s) \Delta s-\int_{T}^{t} \frac{\gamma z(s)}{r^{\frac{1}{\gamma}}(s)}\left(w^{\sigma}(s)\right)^{\frac{1}{\gamma}+1} \Delta s .
$$

Setting $B=z^{\Delta}(s), A=\frac{\gamma z(s)}{r^{\frac{1}{\gamma}}(s)}, \quad u=w^{\sigma}(s)$ and using Lemma 2.3, we get

$$
\int_{T}^{t} z(s) Q_{1}(s) \Delta s \leq z(T) w(T)+\int_{T}^{t} \frac{\gamma^{\gamma}}{(\gamma+1)^{\gamma+1}} \frac{\left(z^{\Delta}(s)\right)^{\gamma+1} r(s)}{\gamma^{\gamma} z^{\gamma}(s)}
$$

and so

$$
\int_{T}^{t}\left[z(s) Q_{1}(s)-\frac{r(s)\left(z^{\Delta}(s)\right)^{\gamma+1}}{(\gamma+1)^{\gamma+1} z^{\gamma}(s)}\right] \Delta s \leq z(T) w(T),
$$

which contradicts condition (30). Then every solution of (1) oscillates. The proof is completed.

Theorem 3.3. Assume that $\left(H_{1}\right)-\left(H_{3}\right)$ and (1) are satisfied. Suppose that $z(t)$ is defined as in Theorem 3.2, $H \in \mathbb{R}$ and for $t>s$,

$\lim _{t \rightarrow \infty} \sup \frac{1}{H\left(t, t_{0}\right)} \int_{T}^{t}\left[H(t, s) z(s) Q_{1}(s)-\frac{r(s) C^{\gamma+1}(t, s)}{(\gamma+1)^{\gamma+1} z^{\gamma}(s) H^{\gamma}(t, s)}\right] \Delta s=\infty$.

is satisfied. Then every solution of (1) oscillates.

Proof. Assume the contrary and let $y$ be a nonoscillatory solution of (1). Without loss of generality, we may assume that

$y(t)>0, \quad y(\tau(t))>0, \quad y(\tau(\tau(t)))>0, \quad y\left(\delta_{i}(t)\right)>0, \quad i=1,2$ for $t \geq T$. (36) Let $w$ be defined as in (11), then $w(t)>0$ for $t \geq T$. Multiplying (32) by $z(t) H(t, u)$ and integrating from $T$ to $t$, we obtain

$$
\int_{T}^{t} H(t, s) z(s) Q_{1}(s) \Delta s \leq-\int_{T}^{t} H(t, s) z(s) w^{\Delta}(s) \Delta s
$$




$$
-\int_{T}^{t} \frac{\gamma z(s) H(t, s)}{r^{\frac{1}{\gamma}}(s)}\left(w^{\sigma}(s)\right)^{\frac{1}{\gamma}+1} \Delta s
$$

for $u=t$. Integration by parts we get

$$
-\int_{T}^{t} H(t, s) z(s) w^{\Delta}(s) \Delta s=H(t, T) z(T) w(T)+\int_{T}^{t}(H(t, s) z(s))^{\Delta} w^{\sigma}(s) \Delta s .
$$

Thus, we have

$$
\begin{aligned}
& \int_{T}^{t} H(t, s) z(s) Q_{1}(s) \Delta s \\
\leq & H(t, T) z(T) w(T)+\int_{T}^{t}\left(H^{\Delta}(t, s) z(s)+H^{\sigma}(t, s) z^{\Delta}(s)\right) w^{\sigma}(s) \Delta s \\
& -\int_{T}^{t} \frac{\gamma z(s) H(t, s)}{r^{\frac{1}{\gamma}}(s)}\left(w^{\sigma}(s)\right)^{\frac{1}{\gamma}+1} \Delta s \\
= & H(t, T) z(T) w(T)+\int_{T}^{t} C(t, s) w^{\sigma}(s) \Delta s-\int_{T}^{t} \frac{\gamma z(s) H(t, s)}{r^{\frac{1}{\gamma}}(s)}\left(w^{\sigma}(s)\right)^{\frac{\gamma+1}{\gamma}} \Delta s .
\end{aligned}
$$

Setting $B=C(t, s), \quad A=\frac{H(t, s) \gamma z(s)}{r^{\frac{1}{\gamma}}(s)}, u=w^{\sigma}(s)$ and using Lemma 2.3, we have

$\int_{T}^{t} H(t, s) z(s) Q_{1}(s) \Delta s \leq H(t, T) z(T) w(T)+\int_{T}^{t} \frac{\gamma^{\gamma}}{(\gamma+1)^{\gamma+1}} \frac{C^{\gamma+1}(t, s) r(s)}{H^{\gamma}(t, s) z^{\gamma}(s) \gamma^{\gamma}} \Delta s$ and

$$
\int_{T}^{t}\left[H(t, s) z(s) Q_{1}(s)-\frac{r(s) C^{\gamma+1}(t, s)}{(\gamma+1)^{\gamma+1} z^{\gamma}(s) H^{\gamma}(t, s)}\right] \Delta s \leq H(t, T) z(T) w(T),
$$

which contradicts condition (30). Then every solution of (1) oscillates. The proof is completed.

\section{Example}

Example 4.1. Let $\mathbb{T}$ be any time scales and we consider the following second order neutral dynamic equation

$$
\left(\left(\left(t y(t)+\frac{1}{2} y\left(\frac{t}{2}\right)\right)^{\Delta}\right)^{\frac{3}{5}}\right)^{\Delta}+\frac{(\sigma(t))^{\frac{3}{5}}}{(2 t-3)^{\frac{1}{5}}} y^{\frac{1}{5}}\left(t-\frac{1}{2}\right)+\frac{(\sigma(t))^{\frac{3}{5}}}{(4 t-5)^{\frac{1}{5}}} y^{\frac{1}{5}}\left(t-\frac{1}{4}\right)=0,
$$

where $t \in[2, \infty)_{\mathbb{T}}$.

$\left(H_{1}\right) \quad \tau(t)=\frac{t}{2} \leq t, \quad \delta_{1}(t)=t-\frac{1}{2}<t, \quad \delta_{2}(t)=t-\frac{1}{4}<t, \lim _{t \rightarrow \infty} \tau(t)=$ $\lim _{t \rightarrow \infty} \delta_{i}(t)=\infty, \quad i=1,2$.

$\left(H_{2}\right) \quad \tau(t)=\frac{t}{2} \in C_{r d}\left(\mathbb{T}, \mathbb{R}^{+}\right), r(t)=1 \in C_{r d}\left(\mathbb{T}, \mathbb{R}^{+}\right)$that $\int_{2}^{\infty} \frac{1}{r^{\frac{1}{\gamma}}(t)} \Delta t=$ $\int_{2}^{\infty} \Delta t=\lim _{a \rightarrow \infty} \int_{2}^{a} \Delta t=\lim _{a \rightarrow \infty} a-2=\infty, m(t)=t \in C_{r d}\left(\mathbb{T}, \mathbb{R}^{+}\right), p(t)=$ $\frac{1}{2} \in C_{r d}(\mathbb{T},[0,1))$ that $m(\tau(t))=\frac{t}{2} \geq 1>\frac{1}{2}=p(t)$ for $t \geq 2$. 
$\left(H_{3}\right) \quad q_{1}(t)=\frac{(\sigma(t))^{\frac{3}{5}}}{(2 t-3)^{\frac{1}{5}}}, \quad \alpha=\frac{1}{5}, \quad q_{2}(t)=\frac{(\sigma(t))^{\frac{3}{5}}}{(4 t-5)^{\frac{1}{5}}}, \quad \beta=\frac{1}{5}, \quad$ that $\quad q_{i}(t) \in$ $C_{r d}\left(\mathbb{T}, \mathbb{R}^{+}\right)$.

Since

$$
\begin{aligned}
Q(t)= & q_{1}(t)\left(\frac{1}{m\left(\delta_{1}(t)\right)}\left[1-\frac{p\left(\delta_{1}(t)\right.}{m\left(\tau\left(\delta_{1}(t)\right)\right)}\right]\right)^{\alpha} \beta^{\alpha}(t, T) \\
& +q_{2}(t)\left(\frac{1}{m\left(\delta_{2}(t)\right)}\left[1-\frac{p\left(\delta_{2}(t)\right.}{m\left(\tau\left(\delta_{2}(t)\right)\right)}\right]\right)^{\beta} \beta^{\beta}(t, T) \\
= & \frac{(\sigma(t))^{\frac{3}{5}}}{(2 t-3)^{\frac{1}{5}}}\left(\frac{1}{t-\frac{1}{2}}\left[1-\frac{1}{2 \cdot \frac{1}{2}\left(t-\frac{1}{2}\right)}\right]\right)^{\frac{1}{5}}\left(\frac{\delta_{1}(t)-T}{t-T}\right)^{\frac{1}{5}} \\
& +\frac{(\sigma(t))^{\frac{3}{5}}}{(4 t-5)^{\frac{1}{5}}}\left(\frac{1}{t-\frac{1}{4}}\left[1-\frac{1}{2 \cdot \frac{1}{2}\left(t-\frac{1}{4}\right)}\right]\right)^{\frac{1}{5}}\left(\frac{\delta_{2}(t)-T}{t-T}\right)^{\frac{1}{5}} \\
= & \frac{(\sigma(t))^{\frac{3}{5}}}{(2 t-3)^{\frac{1}{5}}} \frac{2^{\frac{1}{5}}}{(2 t-1)^{\frac{1}{5}}} \frac{(2 t-3)^{\frac{1}{5}}}{(2 t-1)^{\frac{1}{5}}}\left(\frac{\delta_{1}(t)-T}{t-T}\right)^{\frac{1}{5}} \\
& +\frac{(\sigma(t))^{\frac{3}{5}}}{(4 t-5)^{\frac{1}{5}} \frac{4^{\frac{1}{5}}}{(4 t-1)^{\frac{1}{5}}} \frac{(4 t-5)^{\frac{1}{5}}}{(4 t-1)^{\frac{1}{5}}}\left(\frac{\delta_{2}(t)-T}{t-T}\right)^{\frac{1}{5}}}
\end{aligned}
$$

we get

$$
Q_{1}(t)=Q(t)\left(\frac{r^{\frac{1}{\gamma}}(t) R(t, T)}{r^{\frac{1}{\gamma}}(t) R(t, T)+\mu(t)}\right)^{\alpha} \eta^{\sigma}(t)
$$

where

$$
\left(\frac{r^{\frac{1}{\gamma}}(t) R(t, T)}{r^{\frac{1}{\gamma}}(t) R(t, T)+\mu(t)}\right)^{\alpha}=\left(\frac{t-T}{t-T+\sigma(t)-t}\right)^{\frac{1}{5}}=\left(\frac{t-T}{\sigma(t)-T}\right)^{\frac{1}{5}}
$$

and

$$
\eta^{\sigma}(t)=c_{2}^{\alpha-\gamma}\left(\int_{T}^{\sigma(t)} \frac{1}{r^{\frac{1}{\gamma}}(s)} \Delta s\right)^{\alpha-\gamma}=c_{2}^{-\frac{2}{5}}(\sigma(t)-T)^{-\frac{2}{5}} .
$$

Thus we get

$$
\begin{aligned}
Q(t)\left(\frac{t-T}{\sigma(t)-T}\right)^{\frac{1}{5}}= & \frac{(\sigma(t))^{\frac{3}{5}} 2^{\frac{1}{5}}}{(2 t-1)^{\frac{2}{5}}}\left(\frac{\delta_{1}(t)-T}{t-T}\right)^{\frac{1}{5}}\left(\frac{t-T}{\sigma(t)-T}\right)^{\frac{1}{5}} \\
& +\frac{(\sigma(t))^{\frac{3}{5}} 4^{\frac{1}{5}}}{(4 t-1)^{\frac{2}{5}}}\left(\frac{\delta_{2}(t)-T}{t-T}\right)^{\frac{1}{5}}\left(\frac{t-T}{\sigma(t)-T}\right)^{\frac{1}{5}} \\
> & \frac{(\sigma(t))^{\frac{3}{5}} 2^{\frac{1}{5}}}{2^{\frac{2}{5}} t^{\frac{2}{5}}}\left(\frac{\delta_{1}(t)-T}{\sigma(t)-T}\right)^{\frac{1}{5}}+\frac{(\sigma(t))^{\frac{3}{5}} 4^{\frac{1}{5}}}{4^{\frac{2}{5}} t^{\frac{2}{5}}}\left(\frac{\delta_{2}(t)-T}{\sigma(t)-T}\right)^{\frac{1}{5}} .
\end{aligned}
$$

Archimedes property says if $x$ and $y$ are real numbers with $x>0$, there exists a natural $n$ such that $n x>y$. So, for $t>T$ and $\delta_{i}(t)>T$ there exist constants 
$k_{1}, k_{2}>0$ sufficiently large that

$$
\begin{aligned}
Q_{1}(t) & >\frac{1}{c_{2}^{\frac{2}{5}}(\sigma(t)-T)^{\frac{2}{5}}}\left\{\frac{(\sigma(t))^{\frac{3}{5}} 2^{\frac{1}{5}}}{2^{\frac{1}{5}} t^{\frac{2}{5}}}\left(\frac{t}{k_{1} \sigma(t)}\right)^{\frac{1}{5}}+\frac{(\sigma(t))^{\frac{3}{5}} 4^{\frac{1}{5}}}{4^{\frac{1}{5}} t^{\frac{2}{5}}}\left(\frac{t}{k_{2} \sigma(t)}\right)^{\frac{1}{5}}\right\} \\
& >\frac{(\sigma(t))^{\frac{3}{5}} 2^{\frac{1}{5}}}{2^{\frac{1}{5}} t^{\frac{2}{5}}}\left(\frac{t}{k_{1} \sigma(t)}\right)^{\frac{1}{5}} \frac{1}{c_{2}^{\frac{2}{5}}(\sigma(t))^{\frac{2}{5}}}+\frac{(\sigma(t))^{\frac{3}{5}} 4^{\frac{1}{5}}}{4^{\frac{1}{5}} t^{\frac{2}{5}}}\left(\frac{t}{k_{2} \sigma(t)}\right)^{\frac{1}{5}} \frac{1}{c_{2}^{\frac{2}{5}}(\sigma(t))^{\frac{2}{5}}} \\
& =\frac{1}{2^{\frac{1}{5}} k_{1}^{\frac{1}{5}} c_{2}^{\frac{2}{5}} t^{\frac{1}{5}}}+\frac{1}{4^{\frac{1}{5}} k_{2}^{\frac{1}{5}} c_{2}^{\frac{2}{5}} t^{\frac{1}{5}}} .
\end{aligned}
$$

Let $z(t)=1$, thus we obtain,

$$
\begin{aligned}
& \lim _{t \rightarrow \infty} \sup \int_{T}^{t}\left[z(s) Q_{1}(s)-\frac{r(s)\left(z^{\Delta}(s)\right)^{\gamma+1}}{(\gamma+1)^{\gamma+1} z^{\gamma}(s)}\right] \\
\geq & \lim _{t \rightarrow \infty} \sup \int_{T}^{t}\left[\frac{1}{2^{\frac{1}{5}} k_{1}^{\frac{1}{5}} c_{2}^{\frac{2}{5}}}+\frac{1}{4^{\frac{1}{5}} k_{2}^{\frac{1}{5}} c_{2}^{\frac{2}{5}}}\right] \frac{1}{s^{\frac{1}{5}}} \Delta s=\infty .
\end{aligned}
$$

According to Theorem 3.2, every solution of (39) is oscillatory.

\section{Acknowledgments}

The authors thank the reviewers for their constructive comments in improving the quality of this paper.

\section{Competing Interests}

The author(s) do not have any competing interests in the manuscript.

\section{REFERENCES}

1. Hale, J. K. (1971). Functional differential equations. In Analytic theory of differential equations (pp. 9-22). Springer, Berlin, Heidelberg.

2. Chen, M., \& Xu, Z. (2007). Interval oscillation of second-order emden-fowler neutral delay differential equations. Electronic Journal of Differential Equations, 2007(58), 1-9.

3. Xu, Z., \& Liu, X. (2007). Philos-type oscillation criteria for EmdenFowler neutral delay differential equations. Journal of Computational and Applied Mathematics, 206(2), 11161126. https://doi.org/10.1016/j.cam.2006.09.012

4. Qin, H., Shang, N., \& Lu, Y. (2008). A note on oscillation criteria of second order nonlinear neutral delay differential equations. Computers \& Mathematics with Applications, 56(12), 2987-2992. https://doi.org/10.1016/j.camwa.2008.09.004

5. Hilger, S. (1990). Analysis on measure chainsa unified approach to continuous and discrete calculus. Results in Mathematics, 18(1-2), 18-56. https://doi.org/10.1007/BF03323153

6. Hilger, S. (1988). Ein makettenkalkl mit anwendung auf zentrumsmannigfaltigkeiten. (Doctoral dissertation). Universitt Wrzburg.

7. Agarwal, R. P., \& Bohner, M. (1999). Basic calculus on time scales and some of its applications. Results in Mathematics, 35(1-2), 3-22. https://doi.org/10.1007/BF03322019

8. Bohner, M., \& Peterson, A. (2001). Dynamic Equations on Time Scales: An Introduction with Applications. Birkhuser, Boston 2001. 
9. Zhang, S. Y., \& Wang, Q. R. (2012). Oscillation Criteria for Second-Order Nonlinear Dynamic Equations On Time Scales. Abst. and Appl. Analysis 2012, Article ID 743469. http://dx.doi.org/10.1155/2012/743469

10. Erbe, L., Hassan, T. S., \& Peterson, A. (2009). Oscillation criteria for nonlinear functional neutral dynamic equations on time scales. Journal of Difference Equations and Applications, 15(11-12), 1097-1116. https://doi.org/10.1080/10236190902785199

11. Saker, S. H. (2011). Oscillation criteria for a second-order quasilinear neutral functional dynamic equation on time scales. Nonlinear Oscillations, 13(3), 407-428. https://doi.org/10.1007/s11072-011-0122-8

12. Zhang, S. Y., \& Wang, Q. R. (2015). Oscillation of second-order nonlinear neutral dynamic equations with distributed deviating arguments on time scales. Advances in Difference Equations, 2015(1), 7. https://doi.org/10.1186/s13662-014-0337-y

13. Zhang, S. Y., \& Wang, Q. R. (2010). Oscillation of second-order nonlinear neutral dynamic equations on time scales. Applied Mathematics and Computation, 216(10), 2837-2848. https://doi.org/10.1016/j.amc.2010.03.134

14. Wu, H. W., Zhuang, R. K., \& Mathsen, R. M. (2006). Oscillation criteria for second-order nonlinear neutral variable delay dynamic equations. Applied Mathematics and Computation, 178(2), 321-331. https://doi.org/10.1016/j.amc.2005.11.049

15. Saker, S. H. (2011). Oscillation criteria for a second-order quasilinear neutral functional dynamic equation on time scales. Nonlinear Oscillations, 13(3), 407-428. https://doi.org/10.1007/s11072-011-0122-8

16. Saker, S. H., \& OŔegan, D. (2011). New oscillation criteria for second-order neutral functional dynamic equations via the generalized Riccati substitution. Communications in Nonlinear Science and Numerical Simulation, 16(1), 423-434. https://doi.org/10.1016/j.cnsns.2009.11.032

17. Thandapani, E., \& Piramanantham, V. (2016). Oscillation criteria of second order neutral delay dynamic equations with distributed deviating arguments. Electron. J. Qual. Theory Differ. Equ.61, 1-15. https://doi.org/10.14232/ejqtde.2010.1.61

18. Candan, T. (2011). Oscillation of second-order nonlinear neutral dynamic equations on time scales with distributed deviating arguments. Computers 83 Mathematics with Applications, 62(11), 4118-4125. https://doi.org/10.1016/j.camwa.2011.09.062

19. Candan, T. (2013). Oscillation criteria for second-order nonlinear neutral dynamic equations with distributed deviating arguments on time scales. Advances in Difference Equations, 2013(1), 112. https://doi.org/10.1186/1687-1847-2013-112

20. Saker, S. H., Agarwal, R. P., \& OŔegan, D. (2007). Oscillation results for second-order nonlinear neutral delay dynamic equations on time scales. Applicable Analysis, 86(1), 1-17. https://doi.org/10.1081/00036810601091630

21. Saker, S. H. (2009). Hille and Nehari types oscillation criteria for second-order neutral delay dynamic equations. Dyn. Contin. Discrete Impuls. Syst., Ser. B, Appl. Algorithms, $16(3), 349-360$.

22. Akin-Bohner, E., Bohner, M., \& Saker, S. H. (2007). Oscillation criteria for a certain class of second order Emden-Fowler dynamic equations. Electron. Trans. Numer. Anal, 27, 1-12.

23. Zhang, S. Y., \& Wang, Q. R. (2014). Interval oscillation criteria for second-order forced functional dynamic equations on time scales. Discrete Dynamics in Nature and Society, 2014, Article ID 684068. http://dx.doi.org/10.1155/2014/684068

24. Saker, S. H., OŔegan, D., \& Agarwal, R. P. (2008). Oscillation theorems for secondorder nonlinear neutral delay dynamic equations on time scales. Acta Mathematica Sinica, English Series, 24(9), 1409-1432. https://doi.org/10.1007/s10114-008-7090-7

25. Mishra, L. N. (2017). On existence and behavior of solutions to some nonlinear integral equations with Applications. National Institute of Technology, Silchar, 788(010). 
26. Vandana, Dubey, R., Deepmala, Mishra, L. N., \& Mishra, V. N. (2018). Duality relations for a class of a multiobjective fractional programming problem involving support functions. American Journal of Operations Research, 8(04), 294-311. https://doi.org/10.4236/ajor.2018.84017

27. Yang, X. M., Yang, X. Q., Teo, K. L., \& Hou, S. H. (2005). Second order symmetric duality in non-differentiable multiobjective programming with F-convexity. European Journal of Operational Research, 164(2), 406-416. https://doi.org/10.1016/j.ejor.2003.04.007

28. Mishra, V. N., \& Mishra, L. N. (2012). Trigonometric approximation of signals (functions) in $L_{p}(p \geq 1)$-norm. International Journal of Contemporary Mathematical Sciences, 7(19), 909-918.

29. Deepmala, A. (2014). Study on Fixed Point Theorems for Nonlinear Contractions and its Applications (Doctoral dissertation, Ph. D. Thesis). Pt. Ravishankar Shukla University, Raipur 492 010, Chhatisgarh, India.

Merve Zingil

Department of Mathematics, Faculty of Science, Ege University, 35100 Bornova, Izmir, Turkey.

e-mail: mervezingil@hotmail.com

Fatma Serap Topal

Department of Mathematics, Faculty of Science, Ege University, 35100 Bornova, Izmir, Turkey.

e-mail: f.serap.topal@ege.edu.tr 